\title{
СОВРЕМЕННЫЙ КОМПЛЕКСНЫЙ ПОДХОД К ДИАГНОСТИКЕ ИНСИДЕНТАЛОМ НАДПОЧЕЧНИКОВ В УСЛОВИЯХ КЛИНИКО-ДИАГНОСТИЧЕСКОГО ЦЕНТРА
}

\author{
Прилепина Е.В.
}

Муниципальное бюджетное учреждение здравоохранения Клинико-диагностический чентр «Здоровье», Ростова-на-Дону

ЦЕЛЬ: выявление гормонально-активных опухолей надпочечников и уточнение степени злокачественности новообразований с последующим определением показаний к адреналэктомии.

МАТЕРИАЛЫ И МЕТОДЫ: в период с 1.2017 г. по 12.2018 г. обследованы 297 пациента, которых можно разделить на две группы: 1 группа - 144 пациента (48,5\%) - с инсиденталомами надпочечников без клинической симптоматики, 2 группа - 153 пациента (51.5\%) - с инсиденталомами надпочечников со злокачественной артериальной гипертензией, резистентной к гипотензивной терапии. Для исследования гормональной активности опухолей надпочечников были использованы клинические рекомендации, согласно которым пациентам обеих групп были выполнены следующие лабораторные показатели - фракционированные метанефрины в суточной моче, утренний кортизол плазмы на фоне подавляющего теста с 1 мг дексаметазона и/или уровень кортизола в суточной моче, при наличии артериальной гипертензии - альдостерон-рениновое соотношение. Топическая диагностика опухолей надпочечников заключалась в проведении ультразвуковых методов исследований, компьютерной томографии с использованием контрастного вещества, позитронно-эмиссионной томографии, в результате которых оценивался размер, плотность, качественные характеристики опухоли, а также описание вторичных изменений органа, с помощью которых оценивался злокачественный потенциал инсиденталом надпочечников.

РЕЗУЛЬТАТЫ И ОБСУЖДЕНИЕ: пациентам обеих групп был выполнен комплекс диагностических мероприятий, включающий лабораторные и инструментальные методы исследований, в результате которых выявлены различные опухоли надпочечников. В обеих группах в большинстве случаев обнаружены аденомы надпочечников без гормональной активности: 134 пациента (93\% случаев) в 1 группе и 133 пациента (87\% случаев) во 2 группе, которые требовали динамического наблюдения. Гормонально - активные образования надпочечников, такие как феохромоцитома и кортикостерома, в обеих группах выявлены в равных соотношениях: в 1 группе - у 4 пациентов (2,8\%) обнаружена феохромоцитома, у 2 пациентов $(1,4 \%)$ - кортикостерома, во 2 группе - 5 (3,2\%) и 2 (1,3\%) случая соответственно. Во второй группе обследуемых у 2 пациентов (1,3\%) выявлена альдостерома, которая требовала дальнейшего дообследования в специализированном стационаре. АКТГ - независимый субклинический синдром гиперкортицизма выявлен в обеих группах: 4 пациента (2,8\%) в 1 группе и 11 пациентов (7,2\%) во 2 группе. Опухоли с высоким злокачественным потенциалом с подозрением на адренокортикальный рак и метастазы злокачественных образований других органов выявлены в 4 случаях (2.8\%) в 1 группе обследованных и в 3 случаях (2\%) во 2 группе пациентов. В результате проведенной работы выявлены показания к оперативному лечению у 22 пациентов из обеих групп в равном соотношении (12 из 1 группы и 10 из 2 группы обследуемых). Данной группе больных выполнена роботассистированная адреналэктомия.

Выводы: комплексный подход в диагностике инсиденталом надпочечников вне зависимости от наличия клинической симптоматики у пациентов с использованием современных лабораторных и лучевых методов исследования имеет высокую информативность, помогает сформулировать мнение о диагнозе и тактике ведения пациентов. 